\title{
Influencia de las alteraciones estéticas dentales en la autoestima de los estudiantes del quinto año de secundaria de las instituciones educativas estatales de Pampa Inalámbrica, distrito de Ilo, 2009
}

\author{
Influences of the aesthetic dental alterations in the self-esteem \\ of the fifth degree students of secondary of the institutions \\ educational state of Pampa Inalámbrica, district of Ilo, 2009
}

Víctor J. Loaiza Vela*

http://dx.doi.org/10.21503/CienciayDesarrollo.2010.v11.09

\section{RESUMEN}

Hoy por hoy, las MYPEs comercializadoras necesitan un cambio de estrategia, centrada en los clientes y ya no sólo en los productos, por dos razones: los productos de calidad estándar y el mercado amplio globalizado; por tanto, necesitan adoptar una estrategia centrada en el cliente, pero en el mercado no existen herramientas tecnológicas adecuadas a las MYPEs, que sean de fácil uso, de bajo costo y que integren técnicas de bussines intelligence que les ayuden a adoptar esta nueva estrategia; ya que sólo cuentan con sistemas transaccionales de compras y ventas.

Palabras clave: alteraciones estéticas, autoestima, estética dental.

\section{ABSTRACT}

MYPEs needs a strategy change, centered in the clients and no longer only in the products, for two reasons: the standard products of quality and the market wide globalizado; so, they need to adopt a strategy centered in the client, but in the market technological appropriate tools don't exist to MYPEs that are user-friendly, of low cost and that they integrate technical of bussines intelligence that help them to adopt this new strategy; since they only have transactional systems of purchases and sales.

Words key: aesthetic alterations, self-esteem, dental aesthetics.

\section{INTRODUCCIÓN}

Siendo la boca uno de los puntos focales de la cara, el estado de salud o enfermedad de sus componentes, su capacidad funcional y su estética pueden influir sobre el bienestar psicológico.

Actualmente, la odontología ocupa un lugar preponderante y no cabe duda que el tercio inferior de la cara: labios, dientes, componentes principales de la sonrisa, con su fuerte valor añadido de salud y juventud, han pasado a ocupar un lugar que no parece que vaya a ser desplazado en breve; además de estar hoy particularmente subrayado por los medios de comunicación y el mundo publicitario que crean modelos sociales que tienen gran influencia en la sociedad.

\footnotetext{
* Docente de la Universidad Alas Peruanas, Filial Arequipa.
} 


\section{Problema de investigación}

\section{Determinación del problema}

Actualmente, existe una alta preocupación por la estética dental, pues el temor al rechazo social por cambios en la estética de los dientes que puedan ser visibles al hablar o sonreír pueden constituir un factor importante para la autoestima y autoconfianza de los estudiantes del quinto año de secundaria; por lo tanto, nuestra investigación permitirá un mayor conocimiento y comprensión del tema por parte de los estudiantes y profesionales.

\section{Objetivos}

Determinar la influencia de las malposiciones dentales en la autoestima de los estudiantes del quinto de secundaria de las I.E. estatales de Pampa Inalámbrica, en Ilo.

Determinar la influencia de las alteraciones del color dental en la autoestima de los estudiantes del quinto de secundaria de las I.E. estatales de Pampa Inalámbrica, en Ilo.

Determinar la influencia de los sistemas dentales en la autoestima de los estudiantes del quinto de secundaria de las I.E. estatales de Pampa Inalámbrica, en Ilo.

$>$ Determinar la influencia de las restauraciones incorrectas en la autoestima de los estudiantes del quinto de secundaria de las I. E. estatales de Pampa Inalámbrica, Ilo.

\section{Hipótesis}

Dado que en la formación de la autoestima intervienen factores biológicos, físicos, personales y socioculturales, es probable que al producirse diferentes alteraciones en la estética dental, estas influyan de manera negativa en los niveles de la autoestima.

\section{MATERIAL Y MÉTODO}

\section{Planteamiento operacional}

\section{Instrumentos}

\section{a. Instrumento documental}

- Inventario de autoestima. Libre de influencias culturales (Culture Free SelfEsteem Inventories - Cfsei - 2 forma AD), James Battle.

- Cuestionario de conocimiento e interés dental del estudiante.

- Ficha de observación clínica intraoral, elaborada sobre la base de los indicadores de investigación, aplicada en forma directa e individual.

\section{Campo de verificación}

\section{Ubicación espacial}

La investigación se llevó a cabo en las aulas de la institución educativa Fe y Alegría y la I.E. Almirante Miguel Grau Seminario de Pampa Inalámbrica, en el distrito de Ilo, con un total de 117 alumnos de secundaria.

\section{RESULTADOS}

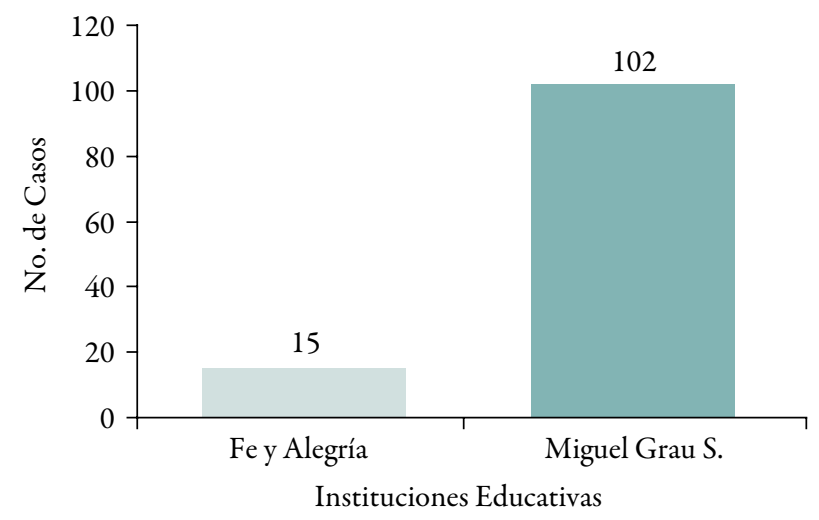

Fuente: Matriz de sistematización.

Figura 1. Distribución numérica de las I.E. estatales de Pampa Inalámbrica, en el distrito de Ilo 
Tabla 1. Distribución numérica y porcentual de las I.E. estatales de Pampa Inalámbrica, en el distrito de Ilo, según edad y sexo

\begin{tabular}{l|cccccc|ccc|cc}
\hline & \multicolumn{6}{|c|}{ EDAD } & \multicolumn{3}{c|}{ SEXO } & \multicolumn{2}{c|}{ TOTAL } \\
INSTITUCIÓN EDUCATIVA & 15 & 16 & 17 & 18 & 19 & 20 & Masculino & Femenino & No & $\%$ \\
\hline FE Y ALEGRÍA & 2 & 5 & 5 & 2 & 0 & 1 & 6 & 9 & $\mathbf{1 5}$ & 13 \\
MIGUEL GRAU & 1 & 57 & 32 & 11 & 1 & 0 & 49 & 53 & $\mathbf{1 0 2}$ & 87 \\
TOTAL & $\mathbf{3}$ & $\mathbf{6 2}$ & $\mathbf{3 7}$ & $\mathbf{1 3}$ & $\mathbf{1}$ & $\mathbf{1}$ & $\mathbf{5 5}$ & $\mathbf{6 2}$ & $\mathbf{1 1 7}$ & $\mathbf{1 0 0}$ \\
\hline
\end{tabular}

Fuente: Matriz de sistematización.

\section{Interpretación}

Según la Tabla 1 y la Figura 1, aceptaron participar de la investigación y cumplieron con los criterios de inclusión 15 alumnos de la institución educativa Fe y Alegría, y 102 alumnos de la institución educativa Almirante Miguel Grau, con un total de 117 alumnos.
En la I.E. Fe y Alegría, 6 son alumnos de sexo masculino y 9 de sexo femenino, representando el $13 \%$ del total de alumnos investigados.

De la I.E. Almirante Miguel Grau son 49 varones y 53 mujeres, con un total de 102 alumnos, que constituyen el $87 \%$ del total del alumnado.

Tabla 2. Relación entre las principales alteraciones estéticas dentales con los diferentes niveles de autoestima

\begin{tabular}{|c|c|c|c|c|c|c|c|c|c|c|c|c|}
\hline \multirow{3}{*}{$\begin{array}{c}\text { ALTERACIONES ESTÉTICAS } \\
\text { DENTALES }\end{array}$} & \multicolumn{10}{|c|}{ DIAGNÓSTICO } & \multirow{2}{*}{\multicolumn{2}{|c|}{ TOTAL }} \\
\hline & \multicolumn{2}{|c|}{ MUY ALTA } & \multicolumn{2}{|c|}{ ALTA } & \multicolumn{2}{|c|}{ PROMEDIO } & \multicolumn{2}{|c|}{ BAJA } & \multicolumn{2}{|c|}{ MUY BAJA } & & \\
\hline & $\mathrm{N}^{\circ}$ & $\%$ & $\mathbf{N}^{\circ}$ & $\%$ & $\mathrm{~N}^{\circ}$ & $\%$ & $\mathbf{N}^{\circ}$ & $\%$ & $\mathbf{N}^{\circ}$ & $\%$ & $\mathbf{N}^{\circ}$ & $\%$ \\
\hline MALPOSICIONES & 7 & $4 \%$ & 24 & $15 \%$ & 51 & $32 \%$ & 59 & $37 \%$ & 19 & $12 \%$ & 160 & $100 \%$ \\
\hline DIASTEMAS & 0 & $0 \%$ & 1 & $4 \%$ & 11 & $44 \%$ & 7 & $28 \%$ & 6 & $24 \%$ & 25 & $100 \%$ \\
\hline ALTERAC. DE COLOR & 0 & $0 \%$ & 4 & $12 \%$ & 12 & $36 \%$ & 12 & $36 \%$ & 5 & $15 \%$ & 33 & $100 \%$ \\
\hline $\begin{array}{l}\text { ALTERACIONES EN LAS } \\
\text { RESTAURACIONES }\end{array}$ & 0 & $0 \%$ & 0 & $0 \%$ & 3 & $38 \%$ & 1 & $13 \%$ & 4 & $50 \%$ & 8 & $100 \%$ \\
\hline OTRAS & 0 & $0 \%$ & 3 & $4 \%$ & 11 & $38 \%$ & 4 & $14 \%$ & 11 & $38 \%$ & 29 & $100 \%$ \\
\hline TOTAL & 7 & 2.7 & 32 & 12.5 & 88 & 34.5 & 83 & 32.5 & 45 & 17.6 & 255 & $100 \%$ \\
\hline
\end{tabular}

Fuente: Matriz de sistematización.

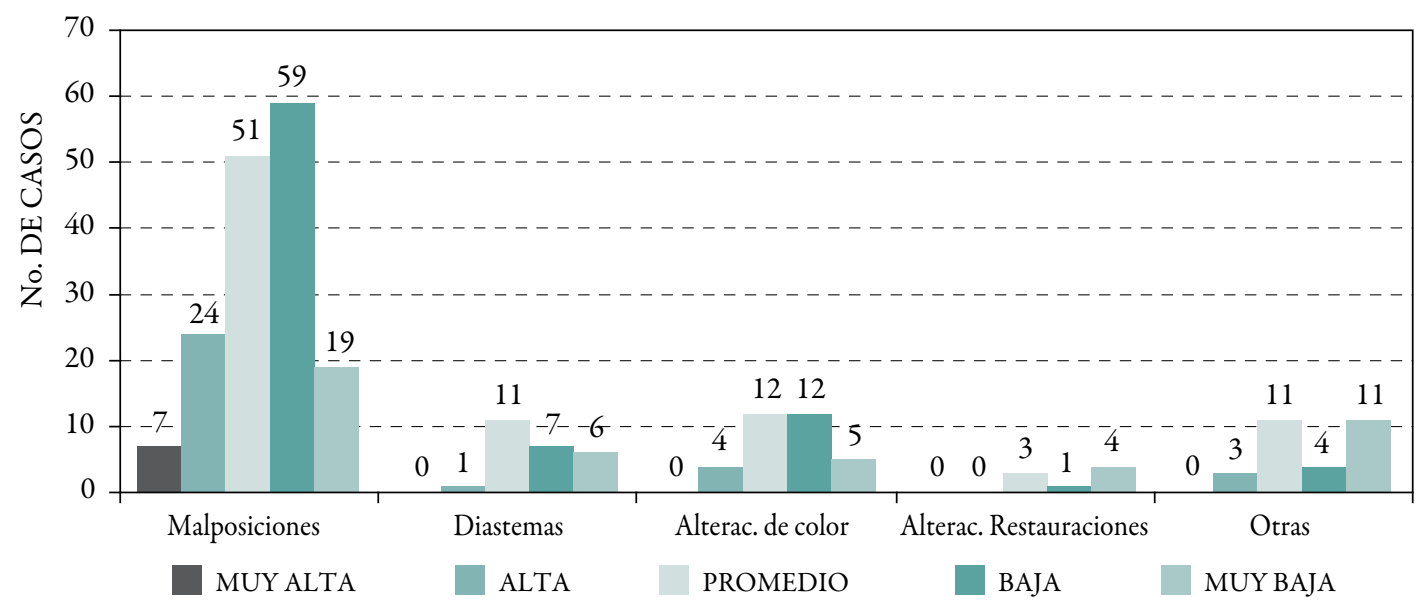

Figura 2. Relación entre las principales alteraciones estéticas dentales con los diferentes niveles de autoestima 


\section{Interpretación}

En la Tabla 2 y la Figura 2 hemos agrupado todas las malposiciones dentales que nos dan una presentación de 160 casos (100\%), de los cuales, 59 casos (37\%) corresponden a autoestima baja, 51 casos (32\%) a autoestima promedio, 24 casos (15\%) a autoestima alta, $19 \operatorname{casos}(12 \%)$ a autoestima muy baja y 7 casos(4\%) a autoestima muy alta.

Los casos que menos se presentaron fueron los de alteración en las restauraciones, con un total de 8 casos, de los cuales 4 (50\%) correspondieron a autoestima muy baja, 3 casos (38 \%) a autoestima promedio y 1 caso (13\%) a autoestima baja.

En total, de los 255 casos de principales alteraciones estéticas dentales presentadas, 88 casos $(34,5 \%)$ corresponden a autoestima promedio, 83 casos $(32,5 \%)$ corresponden a autoestima baja, 45 casos $(17,6 \%)$ a autoestima muy baja, 32 casos (12,5\%) a autoestima alta, y 7 casos $(2,7 \%)$ a autoestima muy alta.

Tabla 3. Distribución del número de alumnos, según nivel de autoestima

\begin{tabular}{l|c|c} 
NIVEL DE AUTOESTIMA & FRECUENCIA & PORCENTAJE \\
MUY ALTA & 5 & $4,27 \%$ \\
ALTA & 15 & $12,82 \%$ \\
PROMEDIO & 41 & $35,04 \%$ \\
BAJA & 41 & $35,04 \%$ \\
MUY BAJA & 15 & $12,82 \%$ \\
TOTAL & $\mathbf{1 1 7}$ & $\mathbf{1 0 0 , 0 0 \%}$
\end{tabular}

Fuente: Matriz de sistematización.

\section{Interpretación}

Según la Tabla 3 y la Figura 3, se observa que la población, en su mayoría, tiene el tipo de autoestima promedio o baja; en ambos casos con $35,04 \%$ y que hacen un total de

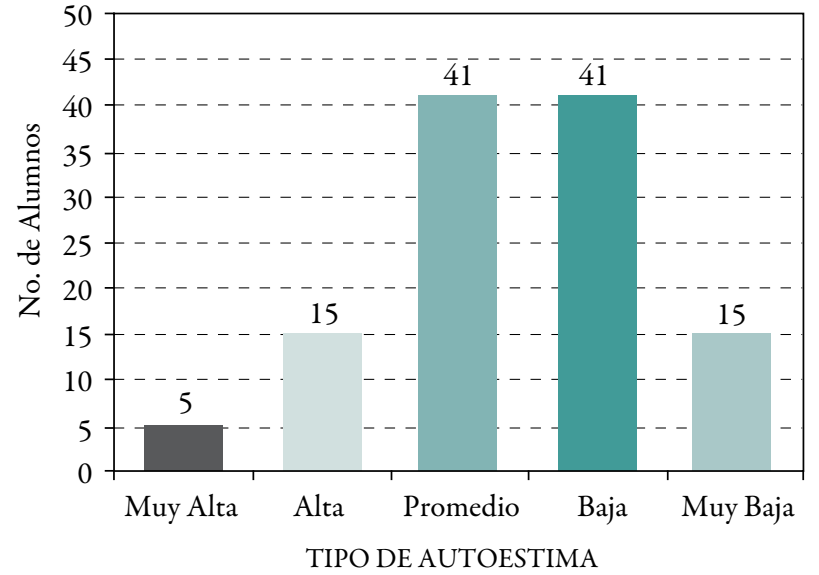

Fuente: Matriz de sistematización.

Figura 3. Distribución del número de alumnos, según nivel de autoestima

$70,8 \%$, siendo un porcentaje significativo de los escolares encuestados. Solamente el 4,27\% tiene autoestima alta.

\section{CONCLUSIONES}

PRIMERA. En el presente estudio se ha determinado que los estudiantes del quinto año de secundaria de las I.E. estatales de Pampa Inalámbrica con alteraciones en la estética dental presentan un nivel de autoestima considerado como promedio y baja, con una inclinación a la autoestima muy baja.

SEGUNDA. Las malposiciones dentarias, así como las alteraciones en el color de los dientes son los principales motivos de los niveles de autoestima promedio, bajo y muy bajo.

TERCERA. 97 alumnos (82,9 \%) tienen autoestima promedio, baja y muy baja, y, según parece, no le otorgan importancia a la estética dental o les es indiferente.

CUARTA. Sobre la hipótesis de que es probable que al producirse diferentes alteraciones en la estética dental, éstas influyan de manera negativa en los niveles de la autoestima, podemos decir que la misma ha sido parcialmente verificada. 


\title{
Obtención de cromato de sodio y óxido de cromo a partir de efluentes industriales
}

\author{
Obtaining of cromato of sodium and chromium oxide \\ starting from industrial efluentes
}

Rosa María Sotomayor Zúñiga*

\section{RESUMEN}

El presente trabajo se plantea la obtención de cromato de sodio y óxido de cromo a partir de los deshechos de sulfato de alumbre que son arrojados al río Chili por las curtiembres de la zona. El sulfato de alumbre es uno de los principales insumos para el desarrollo de la industria curtiembre. Los resultados obtenidos confirmaron la hipótesis de que se puede obtener cromato de sodio y óxido de cromo a partir de los desechos mencionados, reduciendo sensiblemente la contaminación del río Chili, protegiendo así la vida de personas y animales y proveyendo a otras industrias de insumos no contaminantes.

Palabras clave: cromato de sodio, óxido de cromo, efluentes industriales.

\section{ABSTRACT}

The present work thinks about the obtaining of cromato of sodium and chromium oxide starting from those scraps of sulfate that they are heady to the river Chili for the tanneries of the area. The obtained results confirmed the hypothesis that one can obtain cromato of sodium and chromium oxide starting from the mentioned wastes, reducing the contamination of the river Chili, protecting this way the life of people and animals and providing to other industries of inputs non pollutants.

Words key: cromato of sodium, chromium oxide, industrial efluentes.

\section{INTRODUCCIÓN}

La industria de la curtiembre utiliza el cromo en forma de compuestos como el llamado alumbre de cromo, que es el sulfato de alumbre, y cuyo uso es masivo.

Los departamentos de Arequipa y Lima son los principales consumidores del sulfato de alumbre. Después del procesamiento de la curtiembre, los efluentes industriales fueron enviados directa o indirectamente a los ríos de estas ciudades, cuyas aguas presentaron alta concentración de sales de cromo. El río Chili de Arequipa presenta fuerte deterioro por la destrucción de la flora y fauna. La presencia de metales pesados, entre ellos plomo, níquel, mercurio, cromo, etc., nos muestra que las aguas servidas tienen un nivel pobre de oxígeno

\footnotetext{
* Docente de la Escuela Académico-Profesional de Ingeniería Industrial de la Universidad Alas Peruanas, Filial Arequipa.
} 
En la actualidad, la contaminación por cromo no constituye un problema mayor, pero potencialmente existe el peligro por pérdidas directas o indirectas de compuestos químicos, con el cromo como uno de sus componentes.

Es importante considerar la gran actividad química del agua salina, que reacciona con múltiples metales, hasta convertirlos en sales solubles, las cuales pueden ser absorbidas por elementos vivos, ya sea de origen animal o de origen vegetal.

\section{Objetivo}

- Proponer un proceso productivo utilizando tecnologías alternativas para la obtención del cromato de sodio y óxido de cromo.

\section{MATERIAL Y MÉTODO}

Se procedió a tomar muestras del efluente de la curtiembre durante 15 días, con capacidad de 20 litros que contenían en disolución especies de cromo hexavalente y cromo trivalente. Mediante un análisis cualitativo y cuantitativo se encontró que dichos residuos tenían $\mathrm{Cr}(\mathrm{VI})$ y $\mathrm{Cr}$ (III), a partir de los cuales se podían obtener sales de cromato, grado industrial. Las impurezas encontradas fueron principalmente, cloruro de sodio en disolución.

Se desarrolló un proceso (Bazúa-Durán, 1994) mediante el cual se pudieran recuperar en forma de cromato las especies de $\mathrm{Cr}(\mathrm{VI})$.

1. Alimentar al reactor de tanque agitado de acero inoxidable, efluente industrial, previamente filtrado.

2. Agitar constantemente, agregando la potasa $(\mathrm{NaOH})$ en escamas hasta un valor de $\mathrm{pH}$ de 11 (se adicionó en escamas para evitar un incremento en la cantidad de agua).

$\mathrm{H}_{2} \mathrm{CrO}_{4}+2 \mathrm{NaOH} \longrightarrow \mathrm{Na}_{2} \mathrm{CrO}_{4}$ (amarillo) $+\mathrm{H}_{2} \mathrm{O}$

3. Dejar precipitar un sólido de color verde oscuro que contiene las impurezas de cromo trivalente en forma de hidróxidos.

$\mathrm{Cr}^{3+}+\mathrm{OH} \longrightarrow \mathrm{Cr}(\mathrm{OH})_{3}$ (verde oscuro)

4. Filtrar la mezcla anterior para obtener una solución amarilla de cromato de sodio y un sólido residual de color verde.

5. Evaporar la disolución amarilla de cromato hasta su sequedad, para obtener, como producto final, cristales de cromato de potasio.

6. Secar los cristales de cromato de potasio en un horno.

7. Calentar el sólido residual en un horno a $1000{ }^{\circ} \mathrm{C}$, durante 3 a 4 horas, para transformarlo en óxidos diversos y espinelas (cromita), de coloración negra. Estos

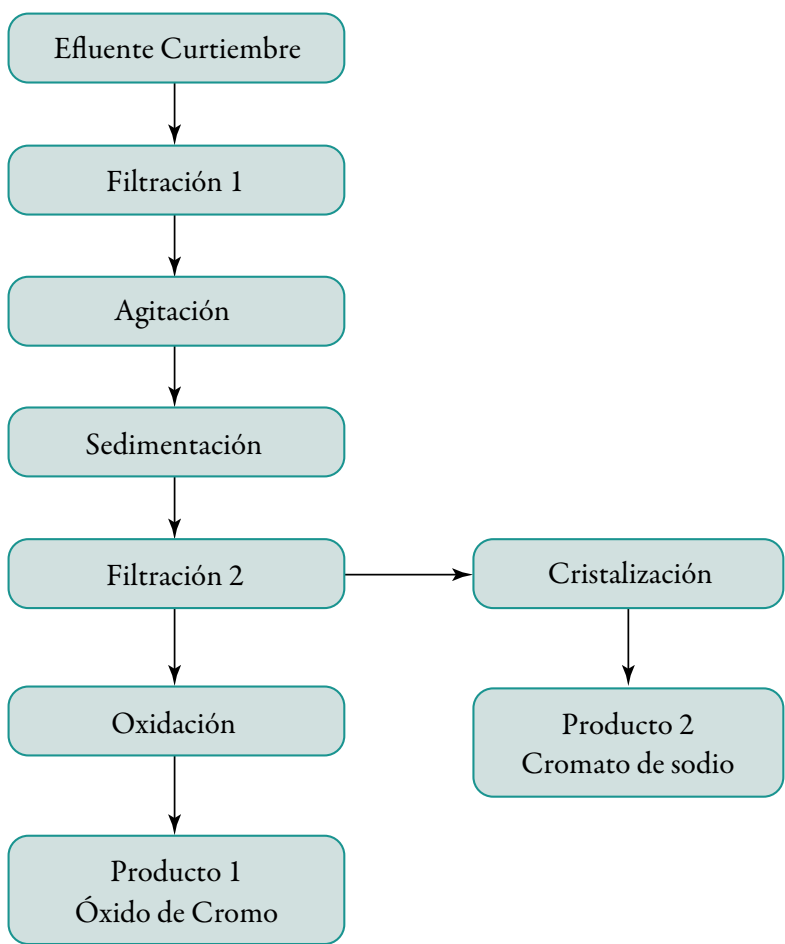

Figura 1. Esquema del proceso 
sólidos son sumamente estables, por lo que pueden ser utilizados como materia prima en la fabricación de ladrillos refractarios o semirrefractarios de cromita.

$2 \mathrm{Cr}(\mathrm{OH})_{3}+$ calor $\rightarrow \mathrm{Cr}_{2} \mathrm{O}_{3}$ (verde) + $3 \mathrm{H}_{2} \mathrm{O}$

\section{RESULTADOS}

Se realizó el análisis cualitativo de tres muestras de los efluentes de curtiembres; los resultados se muestran en la siguiente tabla:

Tabla 1. Resultados del análisis cualitativo

\begin{tabular}{l|c|c|c} 
PARÁMETRO & Muestra 1 & Muestra 2 & Muestra 3 \\
$\mathrm{pH}$ & 3.5 & 3.8 & 3.2 \\
{$\left[\mathrm{CrO}_{4}^{-2}\right]$} & + & + & + \\
{$\left[\mathrm{Cr}^{+3}\right]$} & + & + & + \\
{$\left[\mathrm{Na}^{+1}\right]$} & + & + & + \\
{$\left[\mathrm{Cl}^{-1}\right]$} & + & + & + \\
\hline
\end{tabular}

Una vez que se obtuvieron los productos finales, se realizó el análisis cualitativo y cuantitativo del los productos obtenidos por volumetría para el óxido de cromo, y por espectrofotometría para el dicromato de sodio.

Del efluente industrial se obtuvo el $0,7 \%$ de dicromato de sodio y el $8 \%$ dde óxidos de cromo trivalente.

\section{CONCLUSIONES}

Se logró obtener un proceso tecnológico que trata los residuos altamente tóxicos, transformándolos en cromato de sodio y en óxido de cromo, que es un residuo sólido estable que puede ser utilizado como materia prima para ladrillos refractarios.

\section{REFERENCIAS BIBLIOGRÁFICAS}

1. Arthur I. Voguel. Quimica analitica cualitativa. Kapelusz, Buenos Aires, Argentina. Sexta edición, 1979

2. María del Carmen Bazúa Durán. Reutilización de ácidos de cromo: una metodología aplicable en ámbitos académicos e industriales. Tesis profesional, Facultad de Química, UNAM, México D.F., 1995.

4. Higinio Jacinto Soto. Potencial de contaminación de cromo en el proceso de refinación del petróleo. Tesis profesional, Facultad de Química e Ingeniería Química, UNSM, Lima, 2006. 